\title{
Tumor location and reduction in functional MRI estimates of language laterality
}

\author{
Monika M. Połczyńska, PhD, DLitt, ${ }^{1}$ Lilian Beck, BA, ${ }^{1}$ Taylor Kuhn, PhD, ${ }^{1}$ \\ Christopher F. Benjamin, PhD, ${ }^{2-4}$ Timothy K. Ly, BS, ${ }^{1}$ Kevin Japardi, BA, ${ }^{1}$ Lucia Cavanagh, PhD, \\ and Susan Y. Bookheimer, PhD ${ }^{1}$
}

${ }^{1}$ Department of Psychiatry and Biobehavioral Sciences, David Geffen School of Medicine at UCLA, University of California, Los Angeles, California; and Departments of ${ }^{2}$ Neurology, ${ }^{3}$ Neurosurgery, and ${ }^{4}$ Psychology, Yale University, New Haven, Connecticut

\begin{abstract}
OBJECTIVE Brain tumors located close to the language cortex may distort functional MRI (fMRI)-based estimates of language dominance. The nature of this distortion, and whether this is an artifact of numerous confounders, remains unknown. The authors hypothesized tumor bias based on laterality estimates independent of confounders and that the effects are the greatest for tumors proximal to Broca's area.

METHODS To answer this question, the authors reviewed more than 1113 patients who underwent preoperative fMRI to match samples on 11 known confounders (tumor location, size, type, and grade; seizure history; prior neurosurgery; aphasia presence and severity; and patient age, sex, and handedness). The samples included 30 patients with left hemisphere tumors ( 15 anterior and 15 posterior) and 30 with right hemisphere tumors ( 15 anterior and 15 posterior), thus totaling 60 patients (25 women; 18 left-handed and 4 ambidextrous; mean age 47 [SD 14.1] years). Importantly, the authors matched not only patients with left and right hemisphere tumors but also those with anterior and posterior tumors. Standard fMRI laterality indices (LIs) were calculated using whole-brain and region of interest (ROI) approaches (Broca's and Wernicke's areas).
\end{abstract}

RESULTS Tumors close to Broca's area in the left hemisphere decreased LIs independently of known confounders. At the whole-brain level, this appeared to reflect a decrease in LI values in patients with left anterior tumors compared with patients with right anterior tumors. $\mathrm{ROI}$ analysis replicated these findings. Broca's area LIs were significantly lower $(p=$ 0.02 ) in patients with left anterior tumors (mean LI 0.28) when compared with patients with right anterior tumors (mean LI 0.70). Changes in Wernicke's area-based Lls did not differ as a function of the tumor hemisphere. Therefore, in patients with left anterior tumors, it is essential to assess language laterality using left posterior ROIs. In all remaining tumor groups (left posterior tumors and right hemisphere tumors), language laterality derived from the anterior language ROI was the most robust measure of language dominance.

CONCLUSIONS Patients with tumors close to Broca's area showed more bilateral fMRI language maps independent of known confounders. The authors caution against the assumption that this reduced language laterality suggests no or little risk to language function following tumor resection in the left inferior frontal gyrus. Their results address how to interpret fMRI data for neurosurgical purposes, along with theoretical questions of contralesional functional compensation and disinhibition.

https://thejns.org/doi/abs/10.3171/2020.9.JNS202036

KEYWORDS brain tumor; laterality index; language; fMRI; functional MRI; Broca's area; oncology

A SSESSING which hemisphere is critical (dominant) for language before neurosurgery minimizes new, surgically induced language impairments in patients with brain tumors. ${ }^{1-4}$ While the ways in which brain tumors can alter language network structure and estimates of overall dominance are known, ${ }^{5}$ principles about the relationship between language dominance estimated by functional MRI (fMRI) and tumor location remain unknown.
A fundamental limitation of the valuable work to date has been the absence of well-matched control samples. ${ }^{6-9}$ In studying patients with tumors in the language-dominant hemisphere, an appropriate control would include patients with tumors in similar regions of the nondominant hemisphere ${ }^{7,10}$ who are matched on all other confounders already known to modify estimates of language dominance. Such confounders include, for example, tumor grade,

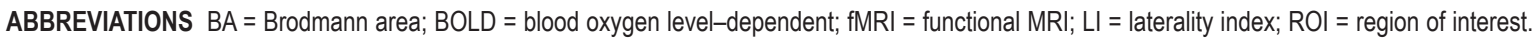

SUBMITTED May 27, 2020. ACCEPTED September 10, 2020.

INCLUDE WHEN CITING Published online April 2, 2021; DOI: 10.3171/2020.9.JNS202036. 
TABLE 1. Missing data summary: number of participants with missing variables

\begin{tabular}{lcccccc}
\hline No. & Variable & Anterior Lt & Posterior Lt & Anterior Rt & Posterior Rt & Total Missing Variables \\
\hline 1 & Tumor location & 0 & 0 & 0 & 0 & 0 \\
\hline 2 & Tumor vol & 0 & 0 & 0 & 0 & 0 \\
\hline 3 & WHO tumor grade & 1 & 2 & 2 & 4 & 9 \\
\hline 4 & WHO tumor type & 1 & 1 & 3 & 1 & 6 \\
\hline 5 & History of seizures & 0 & 1 & 3 & 3 & 7 \\
\hline 6 & Previous surgery & 2 & 1 & 1 & 1 & 5 \\
\hline 7 & Aphasia (yes/no) & 2 & 1 & 0 & 1 & 4 \\
\hline 8 & Aphasia severity & 2 & 1 & 0 & 1 & 4 \\
\hline 9 & Handedness & 0 & 0 & 0 & 0 & 0 \\
\hline 10 & Sex & 0 & 0 & 0 & 0 & 0 \\
\hline 11 & Age & 0 & 0 & 0 & 0 & 35 of 660 \\
\hline
\end{tabular}

presence of aphasia, the history of seizures, and patient demographics (handedness, age, and sex). ${ }^{11-15}$ These variables need to be considered when interpreting the results of presurgical fMRI. ${ }^{10,16}$ Controlling all the confounders that can affect the estimates of fMRI language dominance is challenging, and, in the context of a failure to do so, previous research on the influence of tumor location on language laterality is markedly inconsistent. ${ }^{5-9}$

The goal of this work was to determine the impact of brain tumor location within the language network on language dominance estimated using presurgical fMRI, independent of other known confounders. We reviewed 1113 patients who underwent presurgical fMRI to obtain samples matched on 11 different factors known to affect language dominance. We thus minimized confounders on our results. Following previous research, ${ }^{5-7}$ we hypothesized tumor bias based on laterality estimates independent of confounders and that the effects are the highest for those proximal to Broca's area.

\section{Methods}

This work follows the STROBE guidelines for observational studies.

\section{Patient Population}

The study was overseen by the University of California, Los Angeles, IRB, and all patients provided written informed consent. Data from 1113 patients who received clinical language fMRI at the University of California, Los Angeles, from 2009 to 2018 were reviewed retrospectively. We included all monolingual English-language speakers who had brain tumors directly affecting anterior and posterior language. Patients with anterior tumors had lesions in the inferior frontal gyrus (Brodmann area [BA] 44, BA 45, and BA 47). Patients with posterior tumors had lesions in the posterior middle and superior temporal gyri, angular gyrus, or supramarginal gyrus (BA 22, BA 39, and BA 40). This information was based on medical record review. We assumed that these tumors could potentially affect language areas. Every patient was screened before fMRI to ensure suitability for the examination. With the excep- tion of 2 cases, the patients included in the study did not have aphasia significant enough to fail the initial screening examination. The examination is designed to assess language production (including oral expression writing) and comprehension (including auditory reception and reading).

All patients were left language dominant, as determined clinically. Specifically, language dominance was based on the clinician's reading of the fMRI, clinical conclusions from cognitive assessments, and (in some instances) direct cortical stimulation, which occurred prior to our data analysis. In all cases, the final clinical determination of language dominance was judged to be of high quality independent of the current study.

Our final sample included 60 patients (35 men and 25 women, age 47 [SD 14.1] years) divided into two groups. The left tumor group (target group) comprised 30 patients with left hemisphere tumors and left language dominance (per fMRI), 15 with anterior and 15 with posterior tumors. The right tumor group (control group) comprised 30 patients with right hemisphere tumors and left language dominance, 15 with anterior and 15 with posterior tumors (Table 1).

We followed an approach applied by Wang and colleagues ${ }^{7}$ who used patients with right hemisphere tumors as a control group (also see Kristo et al. ${ }^{17}$ ). Patients with tumors in the non-language-dominant right hemisphere were demonstrated to have fMRI language activations comparable to those of healthy controls with intact Broca's and Wernicke's areas. Concurrently, these patients are more likely to display brain activities related to the psychological and mental processes that are similar to those observed in patients with dominant left anterior tumors. ${ }^{7}$

\section{Sample Selection Process}

Our initial goal was to match the sample size in the aforementioned groups. The limiting factor was, therefore, the size of the smallest tumor group. Fewer right tumor cases are referred for fMRI, and there were fewer right posterior than right anterior cases. Typically, at our center, patients with brain tumors in the right hemisphere are referred for preoperative fMRI, primarily for motor mapping. We almost always administer language tasks to these 


\section{ANTERIOR TUMORS}

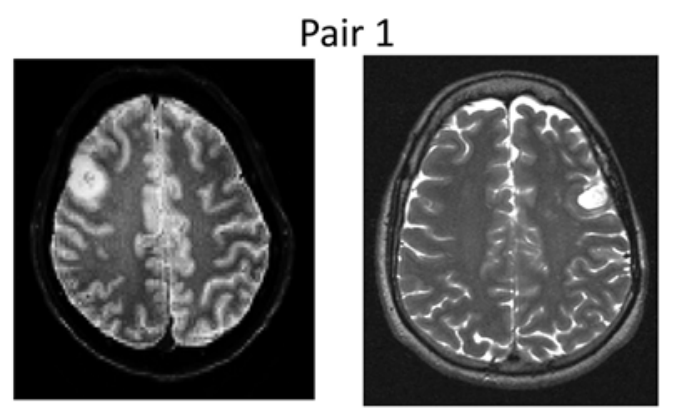

Pair 2

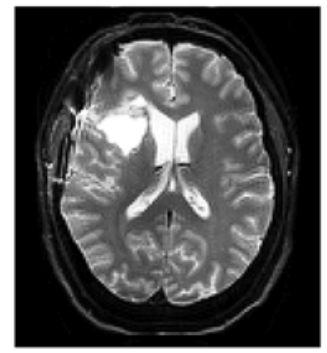

Right hemisphere tumors

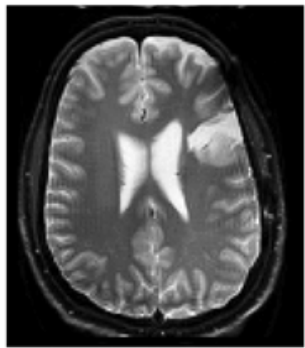

Left hemisphere tumors

\section{POSTERIOR TUMORS}
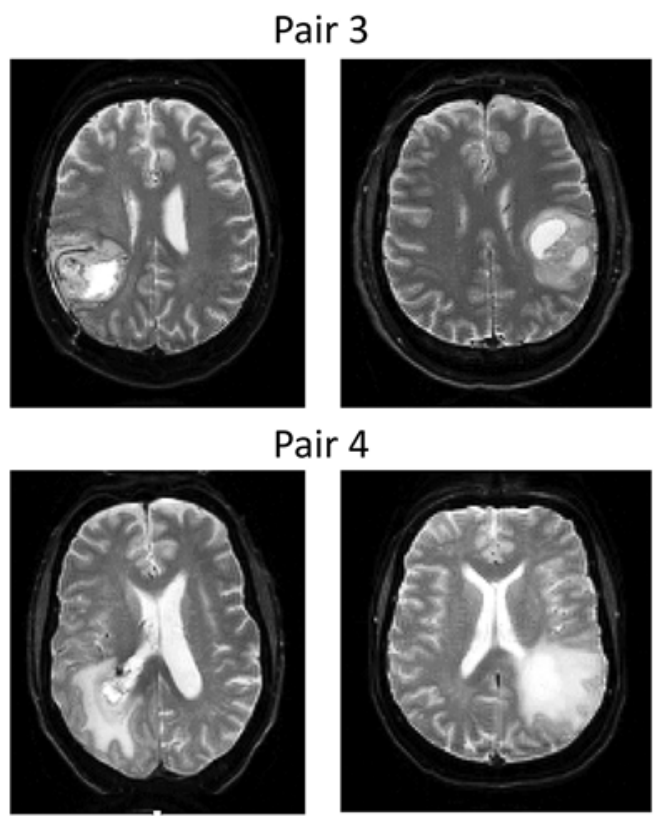

Pair 4

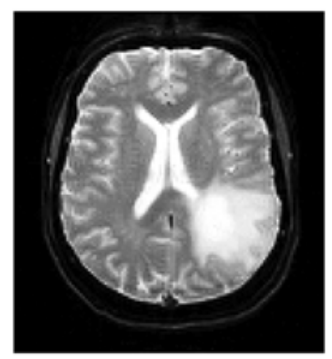

Left hemisphere tumors

FIG. 1. Careful case-by-case patient matching. For each right hemisphere tumor case, we found a visually matched left hemisphere pair based on tumor location and volume. The left tumor cases were additionally matched on the remaining 9 variables known to affect language laterality.

patients. As such, all 15 patients with right posterior tumors were included first. All the right anterior cases were then reviewed to obtain a sample with the closest possible match in terms of the variables below, followed by left anterior and left posterior samples. See Fig. 1 for samples of our case-by-case matching. We only included individuals with brain tumors that directly affected the anterior and posterior language sites. This information was based on medical record review.

\section{Controlled Variables}

We matched our samples based on the following 11 variables: 1) Tumor location: the distance between the tumor and language areas was central to our hypotheses. This distance was matched visually in the right and left hemisphere tumor patients on a case-by-case basis by two independent investigators. 2) Tumor size was also visually matched on a one-to-one basis by two independent investigators. Equivalence was confirmed using tumor masks and lesion heat maps (see Lesion Identification below). 3) Tumor type and 4) tumor grade were defined by the 2016 WHO Classification of Tumors of the Central Nervous System. Here, grade I represents the least malignant tumors and grade IV, the most malignant tumors. Grade I and II tumors are considered low grade, and grade III and IV are considered high grade. ${ }^{18}$ Both variables were defined by record review. Since nearly all of our patients had diffuse astrocytic and oligodendroglial tumors, we also matched our samples based on tumor types within this category (glioblastoma, anaplastic astrocytoma, oli- goastrocytoma, oligodendroglioma). Other clinical factors potentially biasing estimates were identified by record review and included 5) history of seizures and 6) previous surgery. The 7) presence and 8) severity of aphasia were screened at the fMRI session and defined as the neuropsychologist's diagnosis of aphasia in a record review. Demographics, including 9) age, 10) sex, and 11) handedness, were identified based on record review and patient report. Handedness was assessed by asking patients which hand they used to write. The reports were compared with record reviews, and the information was concordant. Missing data are listed in Table 1.

None of these variables differed across groups as a function of either tumor laterality (left vs right) or tumor location (anterior vs posterior) (total comparisons: 20). Two exceptions were that 1) patients with anterior tumors were more likely to have low-grade tumors than patients with posterior tumors $\left(\chi^{2}[1,51]=6.42, \mathrm{p}=0.01\right)$, and 2$)$ patients with posterior tumors were less likely to have aphasia than those with anterior tumors $\left(\chi^{2}[1,56]=4.59, p=0.03\right)$. See Table 2 for details. Continuous variables were analyzed using a univariate one-way ANOVA, and categorical variables were analyzed using the chi-square test.

\section{Procedure}

Patients attended a single session. Before scanning, patients were screened for aphasia with detailed questions regarding language production and comprehension. The 3 fMRI tasks were then practiced, with 3 sample items per 
TABLE 2. Biodemographic information about tumor patients

\begin{tabular}{|c|c|c|c|}
\hline Variable & LH Tumors & RH Tumors & Total \\
\hline No. of patients & 30 & 30 & 60 \\
\hline \multicolumn{4}{|l|}{ Sex } \\
\hline Male & 16 & 19 & 35 \\
\hline Female & 14 & 11 & 25 \\
\hline \multicolumn{4}{|l|}{ Handedness } \\
\hline $\mathrm{Rt}$ & 22 & 16 & 38 \\
\hline $\mathrm{Lt}$ & 7 & 11 & 18 \\
\hline Ambidextrous & 1 & 3 & 4 \\
\hline Mean age, yrs & 47.67 & 46.3 & 47 \\
\hline \multicolumn{4}{|l|}{ Tumor location } \\
\hline Anterior & 15 & 15 & 30 \\
\hline Posterior & 15 & 15 & 30 \\
\hline \multicolumn{4}{|l|}{ WHO tumor grade } \\
\hline | or || & 9 & 11 & 20 \\
\hline III or IV & 18 & 13 & 31 \\
\hline \multicolumn{4}{|l|}{ WHO tumor type } \\
\hline DA\&O tumor & 25 & 26 & 51 \\
\hline Meningioma & 1 & 0 & 1 \\
\hline Metastatic tumor & 0 & 2 & 2 \\
\hline \multicolumn{4}{|l|}{ Seizures } \\
\hline Yes & 18 & 16 & 34 \\
\hline No & 12 & 8 & 20 \\
\hline \multicolumn{4}{|l|}{ Previous surgery } \\
\hline Yes & 15 & 10 & 25 \\
\hline No & 12 & 18 & 30 \\
\hline \multicolumn{4}{|l|}{ Aphasia } \\
\hline Yes & 15 & 15 & 30 \\
\hline No & 12 & 14 & 26 \\
\hline \multicolumn{4}{|l|}{ Type of aphasia } \\
\hline Mild & 8 & 10 & 18 \\
\hline Moderate & 6 & 4 & 10 \\
\hline Severe & 1 & 1 & 2 \\
\hline
\end{tabular}

DA\&O = diffuse astrocytic and oligodendroglial; $\mathrm{LH}=$ left hemisphere; $\mathrm{RH}=$ right hemisphere.

task. Only individuals able to follow task instructions and complete at least some items were included in our study sample. During MRI scanning, patients completed 1 or 2 runs of the 3 language tasks (see below). Two runs were acquired whenever possible. A single run was completed in some cases if time was constrained for other reasons.

\section{Image Acquisition}

Imaging was completed at 3T on a Siemens Allegra or Prisma scanner (12- and 20-channel head coils, respectively). The numbers of patients scanned with each scanner were balanced between the target and control groups. Task echo planar imaging parameters were TE $35 \mathrm{msec}$, TR $2500 \mathrm{msec}, 28$ slices, $90^{\circ}$ flip angle, FOV $200 \mathrm{~mm}^{3}$, voxel size $3.1 \times 3.1 \times 3 \mathrm{~mm}$, and 90 volumes. A matched anatomical T2-weighted image was also acquired.

\section{Functional MRI Tasks}

We map the language network by taking the conjunction of 3 language tasks. Each task uses a different modality to map the language network (e.g., Bookheimer ${ }^{19}$ and Benjamin et al. ${ }^{20}$ ) as follows: 1) Object naming and action generation: patients silently name objects individually presented on the screen and generate an action verb related to the object. ${ }^{21}$ 2) Reading responsive naming: patients silently read written descriptions of objects (e.g., "color of snow") and silently name them. 3) Auditory responsive naming: patients listen to spoken descriptions of objects (e.g., "wool-making animal") and silently name them.22 All use a block design with 10 -second blocks. Blocks began with a 1-second written cue (e.g., "think of the name"), then the task ( 3 trials), and then a crosshair (rest). Each run had 11 task and 12 rest blocks totaling 4 minutes' duration per task.

\section{Analysis \\ Functional MRI Data Analysis}

Our approach to analysis includes minimal preprocessing and identification of the task-related signal using Pearson's correlation coefficient and is described in detail elsewhere. ${ }^{20}$ Briefly, data are smoothed (2-mm gaussian kernel), a regressor including the expected time series is convolved with a hemodynamic response function, and the correlation of actual and expected activity is taken. Data are quality checked and, if two runs were completed, those judged superior were used in the analysis. Each map was thresholded at a correlation of 0.2. This threshold is adjusted until an optimal representation of the known language network is identified. The conjunction of the resulting maps that gives the best representation of the language network is used. This involves an effective significance value of $\mathrm{p}<0.000123\left(0.05^{3}\right)$ and eliminates any activation that is task specific, including sensory activation (e.g., auditory activation in the auditory responsive naming task). This approach is systematic, reliable, and valid when compared with other estimates of language dominance. ${ }^{20}$

\section{Calculation of the Language $\mathrm{LI}$}

We used the thresholded voxel count from the conjunction analysis to evaluate language laterality using 1) the whole brain and 2) the following 4 language regions of interest (ROIs): Broca's area-left BA 44, BA 45, and BA 47; Wernicke's area-left BA 22, BA 39, and BA 40; and the right hemisphere homologs for each. The ROIs were determined functionally. We found in our clinical practice that tasks requiring language production engage more frontal regions (Broca's area), and tasks requiring language comprehension activate more temporal/parietal regions (Wernicke's area). ${ }^{23}$ Task differences in the two areas have also been extensively demonstrated in the literature (e.g., Gaillard et al. ${ }^{22}$ ). Furthermore, since most of the patients who undergo presurgical language fMRI have a brain lesion neighboring Broca's or Wernicke's area, it is helpful to examine these regions separately. ${ }^{6,724} \mathrm{~A}$ standard language laterality index (LI) was calculated ([left - right]/[left + right]) with the value ranging from +1 (left dominance) to -1 (right dominance). ${ }^{25}$ 


\section{Lesion Identification}

On an individual level, we visually matched cases into pairs based on their lesion location (confirmed by medical record review) and size. Borders of individual tumors were based on signal intensity changes on T2-weighted MRI. Using the T2 boundaries, masks of patients' tumors were hand-drawn on patients' T2-weighted MRI scans by a senior investigator using FSL tools. All were reviewed for accuracy by a second clinician/investigator. The lesion included the center of the tumor, any prior resection cavity, and any dense surrounding edema (it is likely that all these lesion characteristics can affect language). Masks were registered to Montreal Neurological Institute (MNI) space (linear registration, $12 \mathrm{df}$ ). Lesion distribution was reviewed by overlaying all lesions within each sample (heat map). Masks were registered to MNI space (linear registration, $12 \mathrm{df}$ ). Tumor volume on a group level was calculated using lesion masks (fslstats). Tumor volume did not differ between 1) the left and right or 2) anterior and posterior tumor groups (t-test, all p <0.05) (Fig. 2).

\section{Evaluation of Study Hypotheses}

Language LI Values and Active Voxels in the Whole Brain. We evaluated the impact of 1) tumor laterality (left vs right) and 2) sagittal location (anterior vs posterior) on language dominance using a series of univariate, one-way ANOVAs. We also assessed the interaction effect between the two variables (hemisphere and location) on language laterality using a two-way ANOVA. An initial model examined the effect of tumor laterality and sagittal location on the LI, through both their main effects and interaction (dependent variable: LI). In a second model, we evaluated how tumor laterality and sagittal location impact the total number of voxels in each hemisphere. In a third, we assessed whether the interaction of tumor laterality and location impacts the total number of active voxels in each hemisphere.

Language LI Values and Active Voxels in ROIs. We evaluated the effect of tumor laterality (left vs right) on LI values in left language-dominant patients by comparing activation in each language area (Broca's and Wernicke's areas) with activation in its right hemisphere homolog using one-way ANOVAs for both 1) anterior and 2) posterior tumor samples. We also conducted one-way ANOVA analysis to compare the number of voxels active during the language tasks in each of the 4 ROIs (Broca's and Wernicke's areas and their right hemisphere homologs) in patients with anterior tumors in the left hemisphere versus right hemisphere and, separately, patients with posterior tumors in the left hemisphere versus the right hemisphere. In addition, we compared voxel counts in Broca's area versus Wernicke's area within each of the 4 patient groups to assess how tumor location affects the fMRI blood oxygen level-dependent (BOLD) signal in language tasks. To assess how many voxels are active in Broca's and Wernicke's areas when it is not affected by a lesion, we obtained activations in these regions from patients with right hemisphere tumors. While doing this on a group level, we admit that there is interindividual variability from patient to patient, which has also been evidenced in healthy volunteers. ${ }^{26}$

\section{Data Availability}

We carefully documented all methods, materials, and data that were used to conduct the research presented in this article, and we agree to share anonymized data on request from any qualified investigator.

\section{Results}

\section{Impact of Tumor Location on Whole-Brain LI Values}

There was no main effect of tumor hemisphere (left vs right) or tumor location (anterior vs posterior) on language LI values when whole-brain activity was examined. There was a significant interaction, however, showing that patients with left anterior tumors had lower language LI values than patients with right anterior tumors $\left[\mathrm{F}(1,59)=5.2, \mathrm{p}=0.03\right.$, partial $\left.\eta^{2}=0.08\right]$. Within the left hemisphere, whole-brain LIs were lower in those with anterior (as compared with posterior) tumors. However, this result was not statistically significant (mean difference 0.19 , SE 0.99, $\mathrm{p}=0.059$; post hoc decomposition analysis) (Fig. 3).

Comparing the number of active voxels during language tasks in the left hemisphere and right hemisphere across our 4 patient groups revealed no significant differences. While not significant, using the hemisphere-location interaction, it was found that patients with an anterior left hemisphere tumor had more active voxels in the right hemisphere than did patients with a right anterior tumor. We observed no significant differences in the number of right hemisphere or left hemisphere voxels between patients with anterior and posterior tumors in the right hemisphere or between patients with tumors in the left hemisphere versus the right hemisphere.

\section{Impact of Tumor Location on ROI LI Values}

The whole-brain findings were largely reproduced when language laterality estimates were examined using an ROI approach. Patients with a brain tumor in the left anterior hemisphere had lower language LIs in Broca's area than did patients with a brain tumor in the anterior right hemisphere $(p=0.020)$. The mean language laterality result in patients with anterior left hemisphere tumors was 0.28 , while the mean language laterality result in patients with anterior right hemisphere tumors was 0.70 . There was no difference in language LI values in Broca's area between patients with a posterior left compared with a posterior right hemisphere tumor. There was no difference in language laterality estimates in Broca's area when patients had anterior versus posterior tumor locations, in the left or right hemisphere. Laterality estimates in Wernicke's area did not differ as a function of the hemisphere or sagittal location (anterior, posterior) of the tumor (all $\mathrm{p}$ $>0.05)$.

The comparison of the total number of voxels activated in each region (rather than LI values) showed no significant difference in voxel counts in Broca's or Wernicke's area when tumors were in any of the regions studied (all p > 0.05; Figs. 4 and 5). While not statistically significant, patients with anterior left hemisphere tumors had considerably fewer active voxels in Broca's area than did the other 3 patient groups. In contrast, there were numerically 

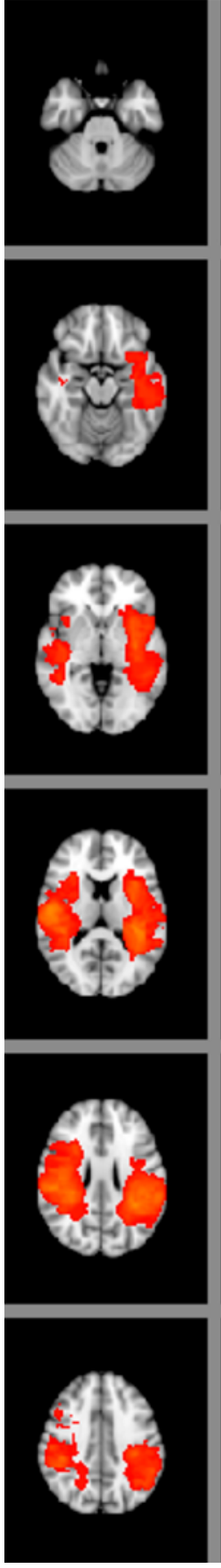
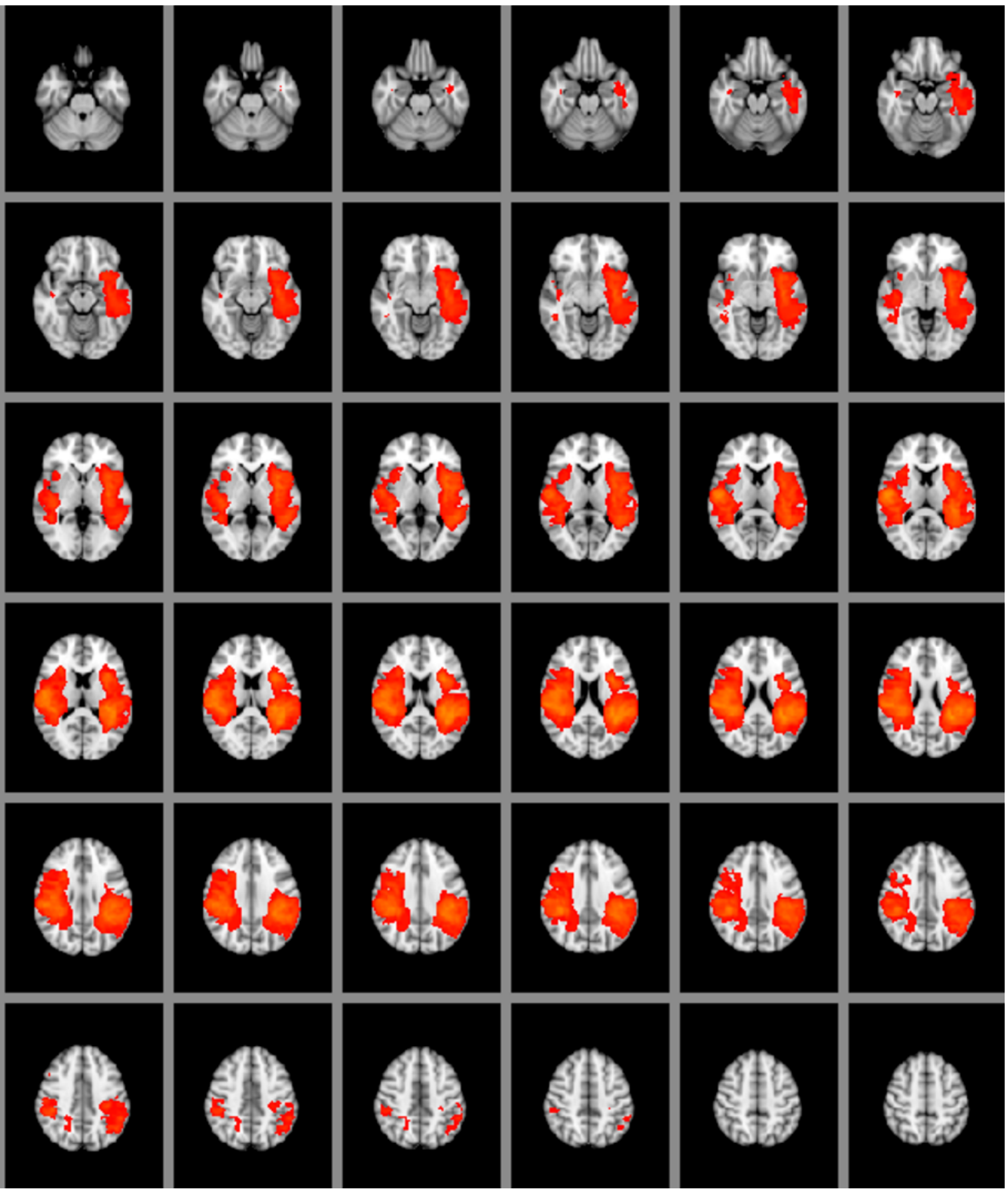

FIG. 2. Tumor heat map showing the locations of left and right brain tumors. We set the minimum number of tumor overlaps to 15 and the maximum number of overlaps to 30 . The left hemisphere is seen on the right side of each brain slice, and the right hemisphere is seen on the left side of each brain slice. Figure is available in color online only.

more active voxels in Broca's area than in Wernicke's area in patients with right anterior hemisphere tumors, in patients with left posterior tumors, and in patients with right posterior tumors (all $\mathrm{p}<0.05$ ).

\section{Discussion}

In this study, we examined the influence of brain tumor location on fMRI estimates of language laterality. Brain tumors located in the left anterior (language-dominant) hemisphere reduced the estimates of language dominance when derived from the signal in Broca's area when all other confounders were controlled. In contrast, estimates based on the fMRI signal in Wernicke's area remained stable even when tumors occurred within posterior language regions. The reduced language laterality occur- 


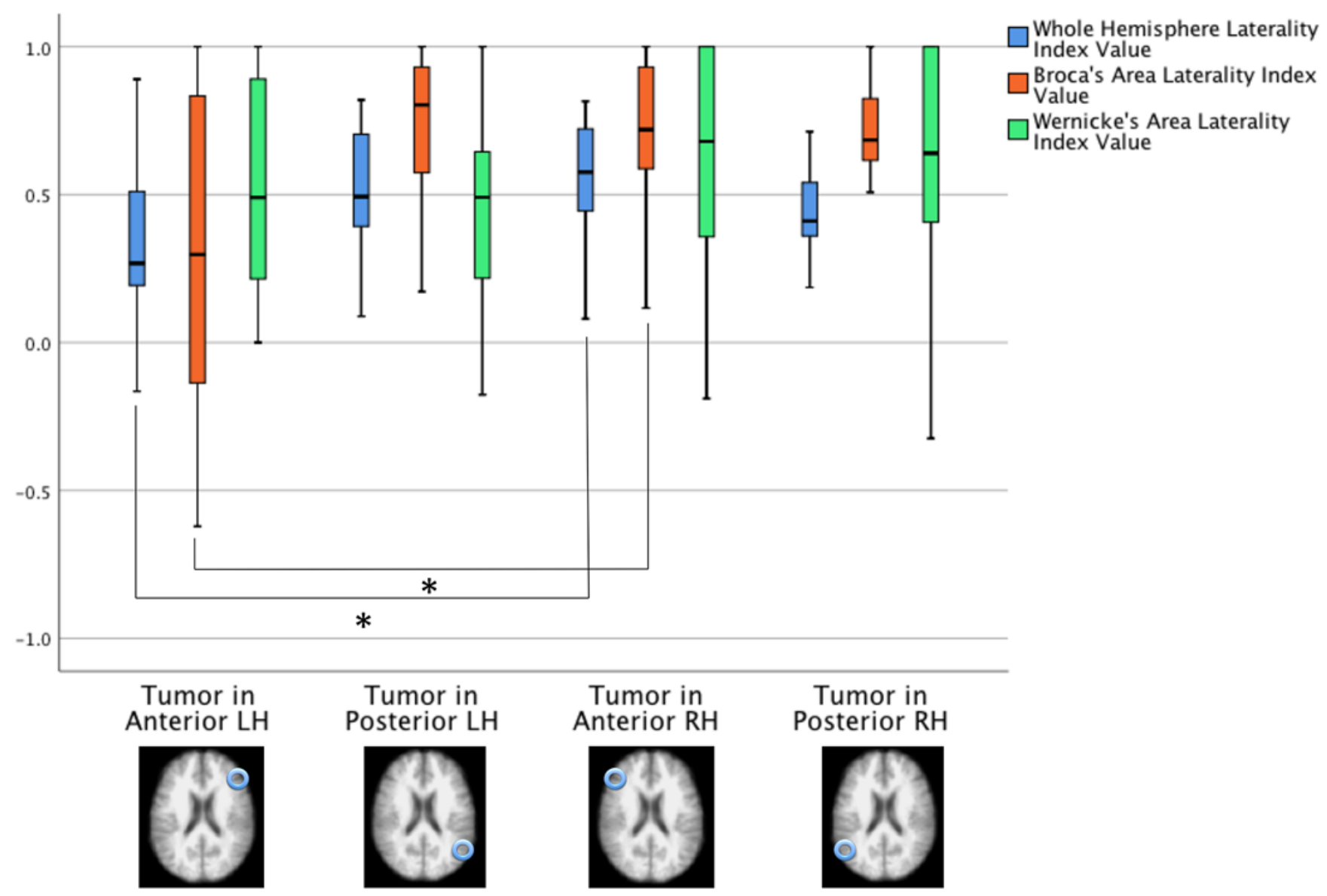

FIG. 3. Language LI values based on the whole-hemisphere approach and an ROI approach. The values were based on $3 \mathrm{fMRI}$ language tasks. Blue bars show language $\mathrm{LI}$ values for language based on active voxel counts in the left hemisphere and right hemisphere. Orange bars illustrate language LI values in Broca's area, and green bars represent language LI values in Wernicke's area. $\mathrm{LH}=$ left hemisphere; $\mathrm{RH}=$ right hemisphere. Figure is available in color online only.

ring with left anterior tumors does not suggest less or no surgery-induced risk to language function after tumor resection. Therefore, in patients with left anterior tumors, it is essential to assess language dominance using posterior language ROIs. In all the remaining tumor groups (left posterior tumors and right hemisphere tumors), language laterality derived from the anterior language ROIs is the most robust measure of language dominance.

In patients with brain tumors, decreased language dominance has been associated with several factors, including weaker activations in language areas proximal to the lesion ${ }^{27}$ and/or increased activity in the nonaffected and non-language-dominant right hemisphere. ${ }^{17,28}$ In the neurosurgical context, it is also critical to understand how language dominance is changed when a tumor lies within the language network. Several prior studies ${ }^{5-9}$ have approached this question, but, to date, results have often proven contradictory.

Consistent with our work, some prior studies have found a relationship between tumor location and language fMRI results. Wang and coauthors ${ }^{7}$ found that patients with left anterior tumors had significantly lower fMRI language dominance than patients with left posterior (and right hemisphere) tumors. Patients with left anterior tumors were more likely, on average, to show right dominance (language $\mathrm{LI}=-0.27$ ). Gohel and colleagues ${ }^{6}$ also observed lower language LIs in patients with left anterior (compared with nonanterior) tumors using resting-state fMRI.

Multiple other studies have observed no consistent relationship. For instance, Nadkarni et al..$^{9}$ found no difference in language laterality between patients with anterior (frontal) and posterior (nonfrontal) lesions. Deng et al. ${ }^{8}$ also found no clear relationship, although they noted a (nonsignificant) trend toward stronger lateralization in left dominant patients with posterior (compared with anterior) tumors.

These apparently contradictory findings likely result from a range of methodological confounders. One is the type of language task used in fMRI, a variable shown to markedly change activation patterns. For instance, Partovi et al..$^{5}$ showed that in patients with tumors affecting Broca's area, language laterality was decreased during a word-generation task but not during sentence generation. Masses in Wernicke's area, however, decreased language laterality estimates based on a sentence-generation task 


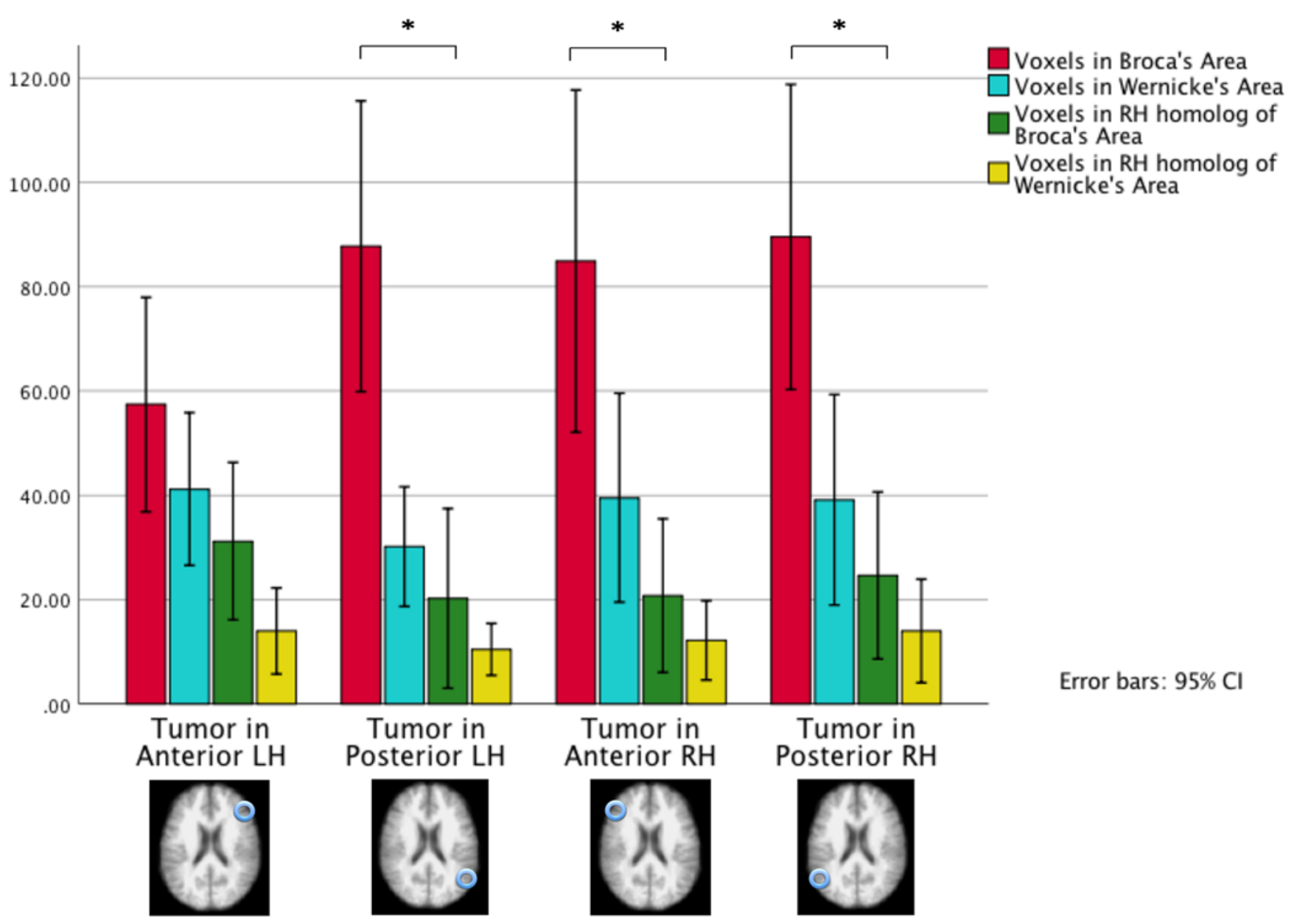

FIG. 4. Active voxel counts in $3 \mathrm{fMRI}$ language tasks in 4 ROls by tumor location. The results were obtained from 4 patient groups that differed based on their tumor location: the anterior right hemisphere, left anterior hemisphere, posterior right hemisphere, and posterior left hemisphere. The 4 ROls are Broca's area (red bars), Wernicke's area (blue bars), the right hemisphere homolog of Broca's area (green bars), and the right hemisphere homolog of Wernicke's area (yellow bars). Patients with an anterior left hemisphere tumor had decreased active voxel counts in Broca's area and slightly increased voxel counts in the right hemisphere homolog of Broca's area. Patients with the left posterior lesions had slightly decreased activity in Wernicke's area. Figure is available in color online only.

but not during word generation..$^{5}$ The tasks used in the aforementioned studies ${ }^{6-9}$ vary from overt picture naming, ${ }^{7}$ silent reading (in Chinese), ${ }^{8}$ and a combination of tasks evaluating language expression (antonym-word generation and letter-word generation) and language comprehension (text-listening and text-reading) ${ }^{9}$ to resting-state fMRI. ${ }^{6}$

Another methodological confounder is variation in the patient groups used across studies. These have varied from solely patients with left hemisphere tumors ${ }^{8,9}$ to the inclusion of patients with both right and left hemisphere tumors. ${ }^{6,7}$ Studies also differ in the use of controls, which have ranged from patients with right hemisphere masses ${ }^{6,7}$ or arteriovenous malformations to groups of healthy individuals. ${ }^{5}$ In some cases, no control group has been used. ${ }^{8}$ Furthermore, previous studies were unable to match cases based on most variables known to affect laterality, including the presence and severity of aphasia, previous brain surgeries, or the history of seizures, tumor grade, tumor volume, sex, and age..$^{5-9}$ The methods used in the current study made it possible to control for these confounders when considering the impact of tumor location on fMRI estimates of language dominance.

Several anatomical and tumor-specific factors may make it more likely for anterior tumors to disrupt laterality estimates. It may take a more extensive lesion to impact language laterality of Wernicke's area than Broca's area, given the region's size; Broca's area is considerably smaller than Wernicke's. ${ }^{29}$ Moreover, the functional organization of Wernicke's (and other) areas appears greater than in Broca's area. ${ }^{29}$ These points suggest that it may be easier to disrupt language function in Broca's area with a smaller brain tumor. Cognitively, the frontal lobe is also centrally involved in the process aiding compensation for language disruption, such as attention, working memory, and executive functioning, ${ }^{30}$ so that an increase in contralateral activation may occur due to the engagement of nonlinguistic process. Consistent with this, we observed a nonsignificant increase in the homolog of Broca's area in 

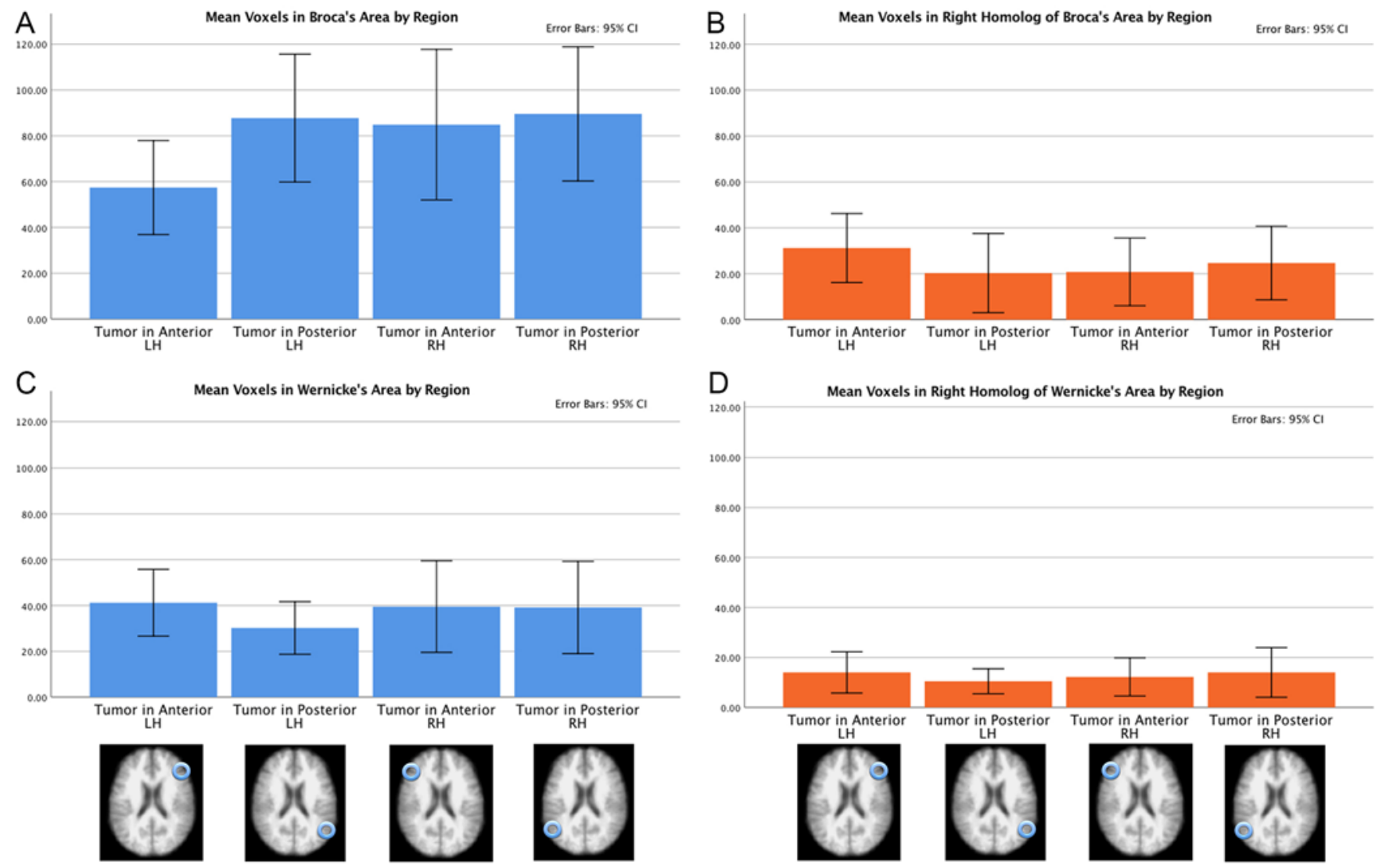

FIG. 5. Active voxel counts in $3 \mathrm{fMRI}$ language tasks in 4 patient groups by ROI. The results were obtained from 4 patient groups that differed based on their tumor location: the anterior right hemisphere, left anterior hemisphere, posterior right hemisphere, and posterior left hemisphere. The 4 ROls are Broca's area (A), the right hemisphere homolog of Broca's area (B), Wernicke's area (C), and the right hemisphere homolog of Wernicke's area (D). In Broca's area, there was decreased activity in the left anterior group. In the right hemisphere homolog of Broca's area, there was slightly increased activity in the left anterior group. In Wernicke's area, there was slightly decreased activity in the left posterior group. The amount of activity in the right hemisphere homolog of Wernicke's area did not differ across the 4 tumor groups. Figure is available in color online only.

anterior left tumor patients but not in Wernicke's homolog in patients with posterior left tumor.

Broca's area is normally a highly lateralizing region. ${ }^{31}$ The difference between the amount of activation in Broca's area and its right hemisphere homolog was robust in all tumor patients in our sample except for the left anterior tumor group. The amount of language activation in Wernicke's area was significantly smaller in the 3 tumor groups, compared with the amount of activity in Broca's area (Fig. 4). Based on this finding, we assume that assessing language laterality based on activity in Broca's and Wernicke's areas taken together may dilute the magnitude of the effect of language laterality in these regions when analyzed separately. The result is in good accord with those of several previous studies. ${ }^{31-34}$ Using task-based fMRI in healthy volunteers, Smitha and colleagues ${ }^{31}$ showed that the BOLD activity in Broca's area was left lateralized in most participants. Conversely, language activity in Wernicke's area was right lateralized or bilateral in half of their patients. ${ }^{31}$

Our results argue against the models of functional compensation and right hemisphere disinhibition (pseudoreorganization). Contralateral disinhibition or functional reorganization would be two possibilities in which language laterality could be reduced with an increase of activation in the right hemisphere. ${ }^{5,16,28,35,36}$ Nevertheless, the absolute numbers in Fig. 3 show that the reason for lower language laterality in the left anterior tumor group was a decreased number of voxels in the left hemisphere and not the slightly increased activity in the right hemisphere. However, no increase in activation occurred in the right homolog of Wernicke's area in the left posterior tumor group. If the activity in the right anterior homologs was caused by disinhibition in both of our left tumor groups, the right hemisphere activity would be focal to the contralateral side of the lesion location, which was not the case in our sample. In contrast, in disorders such as epilepsy, which is a long-standing and slow-growing neurological condition that frequently has an early onset, we may draw different conclusions, including, for example, atypical reorganization of linguistic functions. ${ }^{37,38}$ Nonetheless, brain tumors are commonly later, adult-onset lesions, and most are relatively rapidly growing masses. ${ }^{8}$ In sum, our data did not show a significant change in the right hemisphere that would suggest that contralateral compensation or disinhibition was the primary contributor to reducing 
language laterality in the patients with tumors in the left language-dominant hemisphere.

A number of limitations should be considered when interpreting these findings. The first caveat is the limited size of our anterior and posterior tumor samples $(n=15)$ relative to the hemisphere groups $(\mathrm{n}=30)$. Next, including tumor patients who were left-handed or ambidextrous could be considered a limitation of this study. However, our sample reflects actual clinical referrals, and we, therefore, considered it important to include these patients. Both our left hemisphere versus right hemisphere tumor groups and the anterior versus posterior tumor groups were carefully balanced for handedness distribution. We consider the results of this study directly applicable to the presurgical context in tumor patients (i.e., the results have high external validity). The case-by-case matching explicitly addresses the confounders that have limited the generalizability of prior work. Furthermore, it is a potential weakness that we did not have an independent measure of language laterality in all cases (some patients had electrocorticography, and most patients did not have a Wada test). Another limitation is visual tumor inspection for individual case matching. Nonetheless, in our opinion, visual inspection is necessary because the tumor itself can often distort location, and automated approaches are not going to be effective. Finally, please see the Appendix for additional analyses, ensuring that we minimized bias in our sample. Briefly, we found no significant differences in language LI values between patients with and those without a history of neurosurgery. Our results also did not significantly differ after we removed from our analyses 3 patients with tumors other than diffuse astrocytic and oligodendroglial tumors.

\section{Conclusions}

In this study, we found that the stability of language laterality in Broca's area was disrupted in patients with brain tumors within the left anterior region but not the left posterior language region. The reduced language laterality occurring with left anterior tumors does not suggest less or no surgery-induced risk to language function after tumor resection. Therefore, in these patients, it is essential to assess language laterality using left posterior ROIs. In all remaining tumor groups (left posterior tumors, right hemisphere tumors), language laterality derived from the anterior language ROI was the most robust language dominance measure.

\section{Appendix. Additional Analyses to Ensure Minimal Sample Bias}

It is well known that previous surgery can distort the BOLD signal through signal dropouts and image distortions in areas close to the surgical area. Thus, any actual activation may be obscured, constituting a major confounder in data analysis. This is a problem in the vast majority of patients with previous resections undergoing preoperative fMRI in anticipation of another brain surgery. ${ }^{39}$ One center reported that one-fourth of patients referred for presurgical mapping had a prior resection..$^{40}$ In our sample, $41.7 \%$ of all our patients underwent previous brain surgery, $50 \%$ of the patients had no history of previous surgery, and data were missing for $8.3 \%$ of the patients. As we reported in the Controlled Variables section, the number of patients with a prior resection did not significantly differ in our left and right tumor groups, and between our anterior and posterior samples. We also compared language LI values between patients with and those without surgery and found no significant differences.

Another potentially confounding factor in our study is that there were 3 patients with tumors other than diffuse astrocytic and oligodendroglial tumors (1 meningioma and 2 metastatic tumors). To ensure that the 3 cases did not bias our results, we reran our analyses without these patients. None of the results were significantly different from what we reported when the 3 patients were included in the sample.

The main goal of clinical fMRI also differs substantially from research fMRI analysis in focus on eliminating false negatives rather than false positives. We addressed this issue by using both subjective thresholding (i.e., per individual subject) and conjunction of 3 tasks but realize the standardization of correction remains an ongoing issue in the clinical fMRI field.

\section{Acknowledgments}

We would like to express our gratitude to the reviewers of this manuscript for their insightful comments and suggestions that we have incorporated when revising this manuscript. We also want to thank Huan Wang, Uyen Trinth, and Zachary Nhem for their help with editing the manuscript.

This research has received financial support from the National Institute on Deafness and Other Communication Disorders, grant no. K01DC016904.

\section{References}

1. Kundu B, Penwarden A, Wood JM, et al. Association of functional magnetic resonance imaging indices with postoperative language outcomes in patients with primary brain tumors. Neurosurg Focus. 2013;34(4):E6.

2. Wood JM, Kundu B, Utter A, et al. Impact of brain tumor location on morbidity and mortality: a retrospective functional MR imaging study. AJNR Am J Neuroradiol. 2011;32(8): $1420-1425$.

3. Sabsevitz DS, Swanson SJ, Hammeke TA, et al. Use of preoperative functional neuroimaging to predict language deficits from epilepsy surgery. Neurology. 2003;60(11):1788-1792.

4. Prabhakaran V, Raman SP, Grunwald MR, et al. Neural substrates of word generation during stroke recovery: the influence of cortical hypoperfusion. Behav Neurol. 2007;18(1):45-52.

5. Partovi S, Jacobi B, Rapps N, et al. Clinical standardized fMRI reveals altered language lateralization in patients with brain tumor. AJNR Am J Neuroradiol. 2012;33(11):2151-2157.

6. Gohel S, Laino ME, Rajeev-Kumar G, et al. Resting-state functional connectivity of the middle frontal gyrus can predict language lateralization in patients with brain tumors. AJNR Am J Neuroradiol. 2019;40(2):319-325.

7. Wang L, Chen D, Yang X, et al. Group independent component analysis and functional MRI examination of changes in language areas associated with brain tumors at different locations. PLoS One. 2013;8(3):e59657.

8. Deng X, Zhang Y, Xu L, et al. Comparison of language cortex reorganization patterns between cerebral arteriovenous malformations and gliomas: a functional MRI study. $\mathrm{J} \mathrm{Neu}$ rosurg. 2015;122(5):996-1003.

9. Nadkarni TN, Andreoli MJ, Nair VA, et al. Usage of fMRI for pre-surgical planning in brain tumor and vascular lesion patients: task and statistical threshold effects on language lateralization. Neuroimage Clin. 2014;7:415-423.

10. Fernández-Coello A, Havas V, Juncadella M, et al. Age of language acquisition and cortical language organization in multilingual patients undergoing awake brain mapping. $J$ Neurosurg. 2017;126(6):1912-1923.

11. Szaflarski JP, Binder JR, Possing ET, et al. Language lateral- 
ization in left-handed and ambidextrous people: fMRI data. Neurology. 2002;59(2):238-244.

12. Batouli SAH, Hasani N, Gheisari S, et al. Evaluation of the factors influencing brain language laterality in presurgical planning. Phys Med. 2016;32(10):1201-1209.

13. Nenert R, Allendorfer JB, Martin AM, et al. Age-related language lateralization assessed by fMRI: The effects of sex and handedness. Brain Res. 2017;1674:20-35.

14. Antonsson M, Longoni F, Jakola A, et al. Pre-operative language ability in patients with presumed low-grade glioma. $J$ Neurooncol. 2018;137(1):93-102.

15. Ille S, Engel L, Albers L, et al. Functional reorganization of cortical language function in glioma patients - a preliminary study. Front Oncol. 2019;9:446.

16. Thiel A, Habedank B, Herholz K, et al. From the left to the right: How the brain compensates progressive loss of language function. Brain Lang. 2006;98(1):57-65.

17. Kristo G, Raemaekers M, Rutten GJ, et al. Inter-hemispheric language functional reorganization in low-grade glioma patients after tumour surgery. Cortex. 2015;64:235-248.

18. Louis DN, Perry A, Reifenberger G, et al. The 2016 World Health Organization Classification of Tumors of the Central Nervous System: a summary. Acta Neuropathol. 2016;131(6): 803-820.

19. Bookheimer S. Pre-surgical language mapping with functional magnetic resonance imaging. Neuropsychol Rev. 2007; 17(2):145-155.

20. Benjamin CF, Walshaw PD, Hale K, et al. Presurgical language fMRI: Mapping of six critical regions. Hum Brain Mapp. 2017;38(8):4239-4255.

21. Rutten GJM, Ramsey NF, van Rijen PC, et al. Development of a functional magnetic resonance imaging protocol for intraoperative localization of critical temporoparietal language areas. Ann Neurol. 2002;51(3):350-360.

22. Gaillard WD, Balsamo L, Xu B, et al. fMRI language task panel improves determination of language dominance. $\mathrm{Neu}$ rology. 2004;63(8):1403-1408.

23. Połczyńska MM, Japardi K, Bookheimer SY. Lateralizing language function with pre-operative functional magnetic resonance imaging in early proficient bilingual patients. Brain Lang. 2017;170:1-11.

24. Połczyńska M, Japardi K, Curtiss S, et al. Improving language mapping in clinical fMRI through assessment of grammar. Neuroimage Clin. 2017;15:415-427.

25. Binder JR, Swanson SJ, Hammeke TA, et al. Determination of language dominance using functional MRI: a comparison with the Wada test. Neurology. 1996;46(4):978-984.

26. Omisade A, O'Grady CB, Schmidt MH, Fisk JD. Visual and auditory fMRI paradigms for presurgical language mapping: convergent validity and relationship to individual variables. Neurol Res Int. 2019;2019:6728120.

27. Petrovich NM, Holodny AI, Brennan CW, Gutin PH. Isolated translocation of Wernicke's area to the right hemisphere in a 62-year-man with a temporo-parietal glioma. AJNR Am J Neuroradiol. 2004;25(1):130-133.

28. Ulmer JL, Hacein-Bey L, Mathews VP, et al. Lesion-induced pseudo-dominance at functional magnetic resonance imaging: implications for preoperative assessments. Neurosurgery. 2004:55(3):569-581.

29. Ojemann G, Ojemann J, Lettich E, Berger M. Cortical language localization in left, dominant hemisphere. An electrical stimulation mapping investigation in 117 patients. 1989. $J$ Neurosurg. 2008;108(2):411-421.

30. Fuster JM. Frontal lobe and cognitive development. J Neurocytol. 2002;31(3-5):373-385.

31. Smitha KA, Arun KM, Rajesh PG, et al. Resting-state seedbased analysis: an alternative to task-based language fMRI and its laterality index. AJNR Am J Neuroradiol. 2017;38(6): $1187-1192$
32. Rosazza C, Ghielmetti F, Minati L, et al. Preoperative language lateralization in temporal lobe epilepsy (TLE) predicts peri-ictal, pre- and post-operative language performance: an fMRI study. Neuroimage Clin. 2013;3:73-83.

33. Zhu L, Fan Y, Zou Q, et al. Temporal reliability and lateralization of the resting-state language network. PLoS One. 2014;9(1):e85880.

34. Buklina SB, Batalov AI, Fadeeva LM, et al. The structure of activation of the language zone in patients with intracerebral tumors according to fMRI with respect to tumor location and the functional asymmetry profile. Article in Russian. Vopr Neirokhir. 2015;79(3):60-68.

35. Gębska-Kośla K, Bryszewski B, Jaskólski DJ, et al. Reorganization of language centers in patients with brain tumors located in eloquent speech areas-a pre- and postoperative preliminary fMRI study. Neurol Neurochir Pol. 2017;51(5): 403-410.

36. Traut T, Sardesh N, Bulubas L, et al. MEG imaging of recurrent gliomas reveals functional plasticity of hemispheric language specialization. Hum Brain Mapp. 2019;40(4):1082-1092.

37. Stewart CC, Swanson SJ, Sabsevitz DS, et al. Predictors of language lateralization in temporal lobe epilepsy. Neuropsychologia. 2014;60:93-102.

38. Springer JA, Binder JR, Hammeke TA, et al. Language dominance in neurologically normal and epilepsy subjects: a functional MRI study. Brain. 1999;122(Pt 11):2033-2046.

39. Hua J, Miao X, Agarwal S, et al. Language mapping using T2-prepared BOLD functional MRI in the presence of large susceptibility artifacts -initial results in patients with brain tumor and epilepsy. Tomography. 2017;3(2):105-113.

40. Geißler A, Matt E, Fischmeister F, et al. Differential functional benefits of ultra highfield MR systems within the language network. Neuroimage. 2014;103:163-170.

\section{Disclosures}

The authors report no conflict of interest concerning the materials or methods used in this study or the findings specified in this paper.

\section{Author Contributions}

Conception and design: Połczyńska. Acquisition of data: Cavanagh. Analysis and interpretation of data: Połczyńska, Beck, Benjamin, Ly, Japardi. Drafting the article: Połczyńska. Critically revising the article: Połczyńska, Kuhn, Benjamin, Bookheimer. Reviewed submitted version of manuscript: all authors. Approved the final version of the manuscript on behalf of all authors: Połczyńska. Statistical analysis: Połczyńska, Kuhn. Administrative/technical/material support: Połczyńska, Beck. Study supervision: Połczyńska, Beck.

\section{Supplemental Information}

\section{Previous Presentations}

Portions of this study were presented during an oral slam session and a poster session at the Eleventh Annual Meeting of the Society for Neurobiology of Language, Helsinki, Finland, August 20-22, 2019.

\section{Correspondence}

Monika M. Połczyńska: University of California, Los Angeles, CA.mpolczynska@mednet.ucla.edu. 